\title{
Pelvic Support Hip Reconstruction with Internal Devices: An Alternative to Ilizarov Hip Reconstruction
}

\author{
Sreenivasulu Metikala ${ }^{1}$, Binu T Kurian², Sanjeev S Madan ${ }^{3}$, James A Fernandes ${ }^{4}$
}

\begin{abstract}
Aim and objective: Ilizarov hip reconstruction (IHR) is a traditional method of salvaging chronic adolescent problem hips but faces practical problems from external fixators leading to reduced compliance. We present the same reconstruction utilising only internal devices with a modification in technique and aim to review early results.

Materials and methods: We retrospectively evaluated eight patients between 2014 and 2017 with chronic painful hips treated by a twostage reconstruction; stage 1 included femoral head resection and pelvic support osteotomy using double plating, while stage 2 comprised distal femoral osteotomy avoiding varus followed by insertion of retrograde magnetic nail for postoperative lengthening. Patients continued physiotherapy postoperatively while protecting from early weight-bearing.

Results: At mean follow-up of 19 months (range 6-36), all osteotomies healed with bone healing index of 47 days/cm (range 30-72). Pain improved from 8.3 (range 7-9) to 2 (range 0-6), while limb length discrepancy got corrected from $4.3 \mathrm{~cm}$ (range 3-5) to $1.4 \mathrm{~cm}$ (range 0-2.5) at final follow-up. Trendelenburg sign was eliminated in three and delayed in five. No examples of infection or permanent knee stiffness were noted. One patient had plates breakage due to mechanical fall and one had $35 \mathrm{~mm}$ of lateral mechanical axis deviation requiring corrective osteotomy. Conclusion: Pelvic support hip reconstruction with exclusive internal devices is a technique in evolution with encouraging early results. It avoids common complications of external fixators and facilitates quick rehabilitation of joints. Refraining from distal varus can effectively eliminate Trendelenburg gait, albeit with some degree of lateral mechanical axis deviation. Unlike external fixation where there is a possibility of gradual correction, this staged procedure of internal fixation is technically demanding with a learning curve.

Clinical significance: Pelvic support hip reconstruction performed by internal implants is a viable alternative to IHR with potential benefits. Keywords: Ilizarov hip reconstruction, Internal lengthening nail, Limb lengthening, Pelvic support osteotomy.

Strategies in Trauma and Limb Reconstruction (2020): 10.5005/jp-journals-10080-1459
\end{abstract}

\section{INTRODUCTION}

Hip joints in young adults can be painful, stiff and/or unstable for various reasons, such as, sequelae of septic arthritis, slipped capital femoral epiphysis, developmental dysplasia and so on. Adolescent problem hip, irrespective of its aetiology, may result in chronic disability due to persistent pain, limp, limitation of walking distance and unequal leg lengths. ${ }^{1}$ Additionally, it may lead to a marked Trendelenburg gait, which is both energy inefficient and stressful to the neighbouring joints. ${ }^{2}$ Management, in such situations, is targeted to achieve stable, painless, functional mobile hip with minimal limb length discrepancy (LLD).

Ilizarov hip reconstruction (IHR) has been a conventional salvage with reasonable functional outcomes. ${ }^{3}$ It involves proximal femoral osteotomy for creation of acute valgusextension angulation in conjunction with distal femoral varus osteotomy for realignment of mechanical axis and gradual postoperative lengthening. ${ }^{4}$ The entire procedure is performed by circular external fixators and aims to provide a stable, mobile hip with equal limb lengths and abolish Trendelenburg lurch. Several authors evaluated IHR ${ }^{1,5-9}$ including our case series of 25 selected patients in 2000 to 2012 . However, external fixation devices, in general, have various practical problems. Particularly, when applied to the femur and employed for limb lengthening. The pins and/or wires, inserted through the skin, can create a communicating tract between skin and bone, resulting in pin tract infections and rarely osteomyelitis. Decreased range of adjacent joints' motion can occur due to the impalement of muscles, tendons and fascia. During the prolonged duration, the
1,2Department of Paediatric Limb Reconstruction, Sheffield Children's Hospital, Sheffield, UK

3,4 Department of Paediatric Limb Reconstruction, Sheffield Children's Hospital, Sheffield, UK; Department of Trauma and Orthopaedics, Sheffield Children's Hospital NHS Trust, Sheffield, UK

Corresponding Author: James A Fernandes, Department of Paediatric Limb Reconstruction, Sheffield Children's Hospital, Sheffield, UK; Department of Trauma and Orthopaedics, Sheffield Children's Hospital NHS Trust, Sheffield, UK, e-mail: james.fernandes@nhs.net

How to cite this article: Metikala S, Kurian BT, Madan SS, et al. Pelvic Support Hip Reconstruction with Internal Devices: An Alternative to Ilizarov Hip Reconstruction. Strategies Trauma Limb Reconstr 2020;15(2):91-97.

Source of support: Nil

Conflict of interest: None

external fixator treatment can result in osteopenia, chronic pain and a considerable psychological burden. ${ }^{10}$

The senior author (JAF) proposed a modification of the IHR using entirely internal devices along with a change in surgical technique by avoiding varus at the level of distal osteotomy for the reasons that are explained later (Discussion section). We name it as pelvic support hip reconstruction (PSHR) and is typically executed in two stages. Stage 1 consists femoral head resection, proximal femoral osteotomy and stabilisation by two plates in orthogonal orientation. Stage 2 follows after 2-6 weeks and includes distal femoral osteotomy and insertion of retrograde magnetic nail for 
gradual postoperative lengthening with no varus. We aim to review our early results of PSHR and also compare it with our previous IHR study.

\section{Materials and Methods}

This study was a retrospective evaluation of patients who underwent PSHR at a single academic institution, between July 2014 and September 2017. It was approved by the Ethics and Standard Committee of our institution. Skeletally mature patients with chronic hip pain operated by all-internal PSHR and at least a 6-month follow-up were included. Exclusion criteria consisted of patients operated by a combination of internal and external fixation techniques and inadequate follow-up. A total of eight patients were considered eligible for the study. There were four boys with the mean age of 16 years and four girls with the mean age of 14.33 years. Three patients were the sequelae of the unstable slipped capital femoral epiphysis, two were due to post-septic sequelae, another two belong to post-developmental dysplasia of the hip $(\mathrm{DDH})$ sequelae and the remaining one case was secondary to chemotherapy for acute lymphoblastic leukaemia. All patients had chronic significant hip pain associated with advanced avascular necrosis of femoral head, multiple previous surgeries and some with chondrolysis. As an example, the anteroposterior (AP) radiograph (Fig. 1) of a 17-year-old boy, status post-open surgical dislocation of the left hip and sub-capital realignment for acute unstable slipped upper femoral epiphysis, reveals severe avascular necrosis with degeneration and retained screws. He presented with painful left hip, positive Trendelenburg sign and $5 \mathrm{~cm}$ of shortening. A thorough history was obtained followed by clinical examination assessing the range of motion (ROM) of the hip and knee, Trendelenburg sign and gait, leg length inequality and grading of pain (as per 0-10 numeric rating scale).

\section{Preoperative Planning of Stage 1}

The preoperative planning was based on four standard radiographs including an AP view of the pelvis with both hips, a lateral view of the affected hip with the entire femur, a standing AP mechanical axis radiograph of both lower extremities after equalisation of limb lengths by suitable blocks and, finally, a supine AP radiograph with affected hip in maximum adduction. The overall mechanical axis of

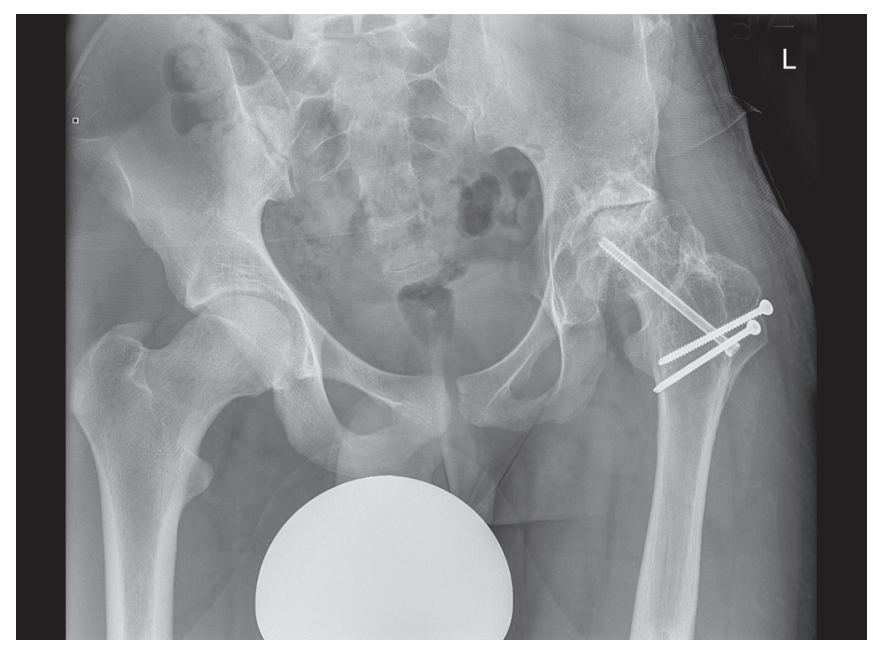

Fig. 1: Pelvic radiograph of an adolescent with secondary osteoarthritis due to avascular necrosis following slipped capital femoral epiphysis and surgical intervention both lower extremities and the respective joint orientation angles were measured in the radiographs. Serum infection marker analysis was performed in all patients to identify active infection. Functional LLD was calculated based on the block test. The point where the femur in maximum adduction coincides with the ischial tuberosity was accepted as the level of the proximal femoral osteotomy and the intraoperative adduction angle. The valgus angle was the outer angle made between the anatomical femoral axis in maximum adduction and a perpendicular line to the horizontal pelvic line (drawn connecting the superior edges of iliac crests or the inferior ends of sacroiliac joints) plus a small overcorrection of $5-10^{\circ}$. The amount of extension was based on the magnitude of flexion deformity but not exceedingly more than $20^{\circ}$. As adduction resulted in external rotation, the entire limb distal to osteotomy was planned to be kept in the maximum internal rotation during internal fixation. In the present study, we created a mean valgus of $41^{\circ}$ (range $30-55$ ) and extension of $15^{\circ}$ (range 10-25).

\section{Preoperative Planning of Stage 2}

A short delay of 2-6 weeks was maintained before the second stage procedure. Patients were mobilised on crutches with no weightbearing on the operated extremity in that interval period. We repeated the AP and lateral radiographs to visualise the full length of the femur including the proximal metalwork. We selected the PRECICE internal magnetic lengthening nail (Ellipse Technologies, Inc., Irvine, California, USA) for stabilisation of osteotomy and gradual postoperative lengthening. All were straight nails with diameter 8.5 or $10.7 \mathrm{~mm}$ based on the width of the distal femur. In light of the proximal plates and screws, the remaining femur segment distal to the terminal screw was considered for calculating the nail length while trying to maintain a gap of one cortical diameter between the ends of the proximal and distal implants. The osteotomy was planned at the distal femur with a goal to maintain the adequate length of the thick segment of the nail in the far segment at the end of the distraction. ${ }^{11,12}$ No varus alignment was planned and lengthening was aimed to progress along a straight anatomical axis.

\section{Surgical Technique}

Pelvic support hip reconstruction, as mentioned earlier, was executed in two stages. Stage 1 was a resection-angulation osteotomy of proximal femur as per the Milch procedure. ${ }^{13}$ Anterior bikini approach was employed to perform the femoral head resection. Careful inspection was performed to identify active infection signs. A separate mid-lateral incision was then utilised for proximal femoral osteotomy and was stabilised by a combination of long and short $3.5 \mathrm{~mm}$ pelvic reconstruction titanium plates in orthogonal fashion (Fig. 2). Benders and pliers were used for the appropriate contouring of the plates. Cancellous bone grafts, harvested from the excised femoral head outside of the collapsed portion, were placed around the osteotomy followed by meticulous closure of the wound.

The second stage of surgery was planned after a delay of 2-6 weeks depending on the patient's general condition and the availability of operation theatre space. The patient was positioned supine and a sterile thigh tourniquet was applied. Retrograde entry into the distal femur was made with the knee in a $30^{\circ}$ flexion. An osteotomy, as decided by the preoperative plan, was performed at the distal femur by a standard low-energy drill hole technique. The capacious medullary canal was prepared with gentle reaming and the selected PRECICE implant was inserted. 


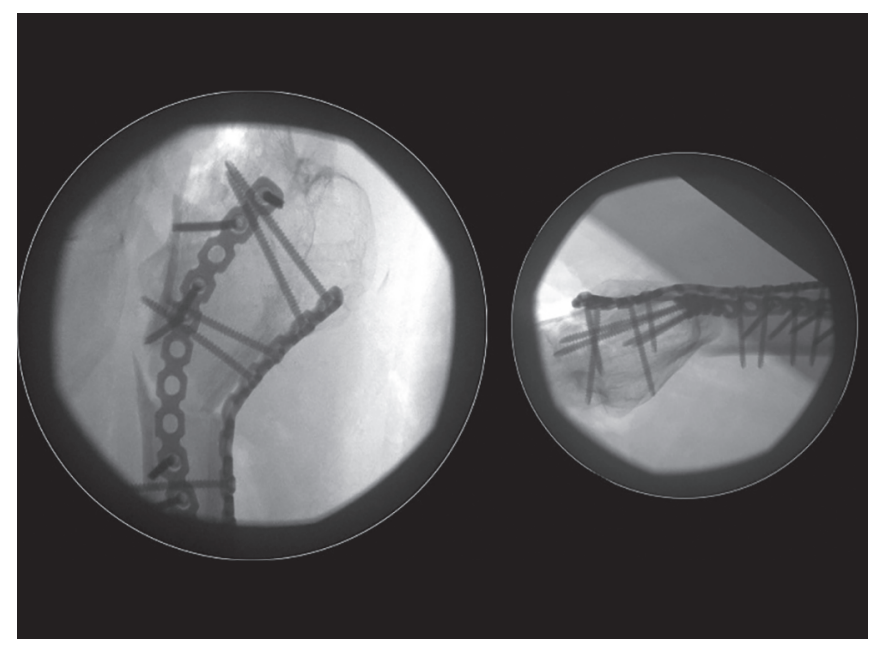

Fig. 2: Fluoroscopic perioperative images showing the valgus extension osteotomy stabilized with double plating internal fixation technique

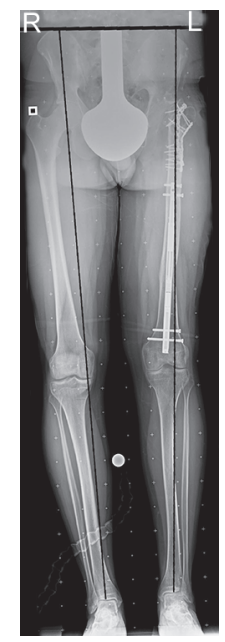

Fig. 4: Final mechanical axis view standing with consolidated regenerated and realigned axis in slight valgus MAD

With the tip of the nail at about $1 \mathrm{~cm}$ distal to the osteotomy drill holes, a sharp osteotome was used to complete the osteotomy. The nail was then advanced across the osteotomy and was locked in a static mode. Fascia lata was generously released and the wounds were closed. External remote control (ERC) was activated to achieve $1 \mathrm{~mm}$ of distraction before transferring the patient out of the operating room.

\section{Postoperative Management}

Ambulation was encouraged from day 1 postoperative with a pair of crutches along with supervised physiotherapy for the adjacent joints. Patients were tutored about the usage of the ERC device. Distraction was commenced a week after surgery at a rate of 1 $\mathrm{mm} /$ day $(0.33 \mathrm{~mm}$, every 8 hours). All were periodically evaluated in the outpatient department, once-weekly during the distraction phase and four-weekly in the consolidation phase. Orthogonal radiographs were obtained at every visit to assess the quality of the regenerate (Fig. 3) and appropriate adjustments were made in the distraction rate. Patients were protected from weight-bearing on the operated extremity until the radiological visualisation of three out of four cortices. Standing mechanical axis radiographs were

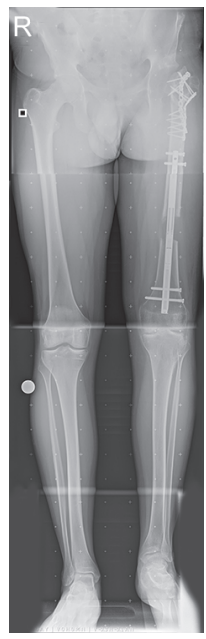

Fig. 3: Standing mechanical axis view with distraction in progress with the intramedullary lengthening nail

repeated at the final follow-up to assess the overall axis (Fig. 4). Removal of all implants was advised between 12 months and 24 months after index surgery.

\section{Results}

The mean follow-up period was 19 months (range 6-36). Outcomes were measured based on the radiological and clinical parameters. Modified mechanical axis line (MMAL), mechanical axis deviation (MAD) and bone healing index (BHI) were measured radiologically. Modified mechanical axis line represents a vertical line ${ }^{1}$ that starts from the horizontal pelvic line (connecting highest points of iliac crests), passes through the proximal osteotomy and extends distally towards the centre of the ankle joint (Fig. 3). Bone healing index represents the number of days before full weight-bearing per centimetre length gain. In other words, it was the period between index operation and full weight-bearing without crutches. The mean MAD, calculated from the knee joint centre to MMAL, was 20 $\mathrm{mm}$ (range 9-35) in a lateral direction. The mean $\mathrm{BHI}$ was 47 days $/ \mathrm{cm}$ (range 30-72) and the mean length gain was $3.5 \mathrm{~cm}$ (range 2.5-5). The clinical evaluation was based on four parameters that include pain during walking and lying down, LLD, hip and knee ROM and Trendelenburg sign. The mean LLD improved from $4.3 \mathrm{~cm}$ (range $3-5 \mathrm{~cm}$ ) preoperatively to $1.4 \mathrm{~cm}$ (range $0-2.5 \mathrm{~cm}$ ). All were positive for Trendelenburg sign before the treatment and three became negative at the time of the final evaluation. The remaining five had delayed Trendelenburg sign and were continuing physiotherapy together with the home exercise program. The pain was evaluated during walking and lying down by 0 to 10 numeric pain rating scale, which showed significant improvement from a mean preoperative value of 8.3 (range 7-9) to 2 (range $0-6$ ). Range of motion was primarily compared for knee flexion, hip flexion and abduction. Three patients, at the final follow-up, showed equal ROM and the remaining five demonstrated less than $20^{\circ}$ reduction of their respective preoperative values. According to a predesigned clinical scoring system, described in Table $1^{8}$ including the aforementioned four clinical parameters, there were 2 (28.5\%) excellent, $3(37.5 \%)$ good, 3 (37.5\%) fair and none showed poor results. No infections or permanent knee stiffness were noted. We observed two complications in our series. One patient fell at 8 weeks post-surgery, breaking both plates at the level of the proximal osteotomy but with no failure of the magnetic nail. Since the radiographs had already 
demonstrated stable callus, the patient was advised protected weight-bearing for an additional month, which resulted in complete healing. One patient had a significant lateral deviation of the mechanical axis by $35 \mathrm{~mm}$ and was recommended a corrective varus osteotomy of the distal femur. A summary of the results is explained in Table 2.

\section{Discussion}

Achieving a functional, stable and pain-free hip in young adults with chronic hip joint pathologies is a challenging task. Ilizarov hip reconstruction has been a routine salvage to equalise limb lengths, eliminate Trendelenburg gait and improve overall biomechanics. We previously evaluated IHR between 2000 and 2012 among 25 young patients with a mean age of 15 years 4 months for various hip pathologies. ${ }^{14,15}$ Ilizarov apparatus was utilised in the first 10 while the subsequent 15 received a hybrid system of proximal llizarov construct and a hexapod frame across the distal osteotomy. All were operated by the classic combination of pelvic support valgus, extension osteotomy in combination with ipsilateral distal femoral osteotomy for lengthening and valgisation aiming to avoid deviation of the mechanical axis. The magnitude of distal varus was decided by the clinical assessment to achieve limb parallelism and

Table 1: Predesigned scoring system ${ }^{8}$

\begin{tabular}{ll}
\hline Overall result & Parameters \\
\hline Excellent & No pain (score 0) \\
& No LLD \\
& ROM equal to or better than before surgery \\
& Negative Trendelenburg sign \\
Good & Mild pain (score 0-3) \\
& LLD $<2.5 \mathrm{~cm}$ \\
& Reduced hip and/or knee ROM $<20^{\circ}$ \\
& Negative or delayed Trendelenburg sign \\
Fair & Moderate pain (score 4-6) \\
& LLD $>2.5 \mathrm{~cm}$ \\
& Reduced hip and/or knee ROM between 20 and 30 \\
& Positive Trendelenburg sign \\
& Continuous and/or severe pain (score 7-10) \\
& LLD $>5 \mathrm{~cm}$ \\
Poor & Reduced hip and/or knee ROM $>30^{\circ}$ \\
& Positive Trendelenburg sign
\end{tabular}

LLD, limb length discrepancy; ROM, range of motion a straight mechanical axis, radiologically. We, however, identified that nearly $30 \%$ of them continued to lurch postoperatively indicating persistent abductor insufficiency. The senior author (JAF) then reasoned out that the distal varus correction countered the benefit of proximal valgus, which was intended for the restoration of abductor tension in addition to neutralising the adduction deformity. As a consequence, it had reintroduced adduction at the hip, thereby retaining the positive Trendelenburg effect. Furthermore, in younger patients, we observed that the proximal osteotomy remodelled much faster than the distal osteotomy which also led to inappropriate excess of overall varus. With all the above reasons, we intentionally avoided the distal varus in the subsequent patient cohort of the IHR group. This change in our surgical technique had successfully abolished the Trendelenburg lurch that series. Coming to the present study of PSHR, with the aforementioned reasons, we had made no attempt of varus alignment in all the eight patients at the level of distal femoral osteotomy and the limb lengthening was achieved along the anatomic axis. It, once again, yielded a similar improvement of the Trendelenburg weakness among all the patients postoperatively but at the expense of lateral deviation of the mechanical axis. The axis deviation, to some extent, can be minimized by conscientiously calculating the overall valgus angle during stage 1 . Hence, the amount of overcorrection, in all eight, did not exceed $10^{\circ}$, contrary to $15^{\circ}$ of overcorrection that was suggested before. ${ }^{1}$ Despite the radiological evidence of lateral MAD, all the extremities were reasonably aligned by clinical examination. However, the effect of lateral MAD at the knee joint needs to be monitored in the long-term. Only one patient had a significant lateral deviation of the mechanical axis by $35 \mathrm{~mm}$ and was recommended for varus correction at the distal femur.

Infection rates also differ considerably between the external and intramedullary devices. The mean fixator time in our IHR group was 173 days and all patients had more than one pin site problem that resolved mostly with local pin site care. Nine patients required oral antibiotics while two patients needed additional courses of parenteral antibiotics. Frank osteomyelitis developed in one patient which was managed by surgical debridement and pin exchange. In literature, up to 1-2 infections per patient were commonly reported with external fixators having an overall incidence of $28-45 \%$ for superficial ${ }^{10,16}$ and up to $23 \%$ for deep infections ${ }^{17}$ requiring surgical attention, such as, debridement, change or removal of pin or wires. On the contrary, no infections were identified in our PSHR group supporting previous studies with $0 \%$ incidence of infection with intramedullary lengthening

Table 2: Summary of results

\begin{tabular}{|c|c|c|c|c|c|c|c|c|c|}
\hline No & Age/gender & $\begin{array}{l}\text { Primary } \\
\text { diagnosis }\end{array}$ & $\begin{array}{l}\text { Lateral MAD } \\
(\mathrm{mm})\end{array}$ & $\begin{array}{l}\text { BHI } \\
\text { (days } / \mathrm{cm} \text { ) }\end{array}$ & $\begin{array}{l}\text { Length gain } \\
(\mathrm{cm})\end{array}$ & $\begin{array}{l}\text { Postoperative } \\
\text { Trendelenburg sign }\end{array}$ & $\begin{array}{l}\text { Follow-up } \\
\text { (months) }\end{array}$ & Complications & $\begin{array}{l}\text { Overall } \\
\text { result }\end{array}$ \\
\hline 1 & 15 years/F & $\mathrm{DDH}$ & 35 & 72 & 2.5 & Delayed & 36 & $\begin{array}{l}\text { Lateral MAD } 3.5 \\
\mathrm{~cm}\end{array}$ & Fair \\
\hline 2 & 16 years $/ M$ & Sepsis & 12 & 44 & 4 & Negative & 20 & None & Excellent \\
\hline 3 & 17 years/M & SUFE & 22 & 60 & 3 & Delayed & 31 & None & Good \\
\hline 4 & 13 years / F & $\mathrm{DDH}$ & 20 & 30 & 3 & Negative & 48 & None & Good \\
\hline 5 & 16 years $/ F$ & Chemotherapy & 22.5 & 46 & 3.5 & Delayed & 26 & None & Fair \\
\hline 6 & 13 years $/ M$ & SUFE & 23 & 44 & 3 & Delayed & 20 & $\begin{array}{l}\text { Plates breakage } \\
\text { at } 8 \text { weeks }\end{array}$ & Fair \\
\hline 7 & 12 years $/ F$ & Sepsis & 16 & 40 & 4 & Negative & 23 & None & Excellent \\
\hline 8 & 17 years $/ M$ & SUFE & 9 & 30 & 5 & Delayed & 12 & None & Good \\
\hline
\end{tabular}

$\mathrm{BH}$, bone healing index; $\mathrm{DDH}$, developmental dysplasia of the hip; MAD, mechanical axis deviation; SUFE, slipped upper femoral epiphysis 
nails. ${ }^{18-21}$ Discussing further between our two studies, the IHR group had one case of nonunion of proximal osteotomy, which was treated with plating and autologous bone grafting. Premature consolidation of regenerate developed in two patients requiring a re-do osteotomy. Finally, permanent knee stiffness was noted in two patients and one had undergone quadricepsplasty while the other patient declined further procedures. Lengthy period, on average of 6 months, in an external femoral circular fixator is a point of concern with several practical problems. Wires and pins passing through the muscles and fascial planes are the main reasons associated with pain, repeated infections, compromised aesthetic look, stiffness of the neighbouring joints and prolonged rehabilitation..$^{10,22,23}$ Overall, according to Paley's classification of difficulties that occur during limb lengthening, ${ }^{10}$ our IHR group had multiple examples of "problems" including pin site infections that resolved with local treatment in the clinic; four events of "obstacles" including one case of osteomyelitis, one case of nonunion of the proximal osteotomy and two patients with premature consolidation of regenerate; and two instances of "complications" consisting of permanent knee stiffness. On the contrary, there were no examples of "problems" and "complications" in the PSHR group, although two "obstacles" were observed including plates breakage and excessive axis deviation with one in each category. The comparison between IHR and PSHR studies performed at our institution is summarised in Table 3.

Internal fixation of pelvic support osteotomy by plating was previously described for neglected congenital/neuromuscular hip dislocations and post-septic ankyloses of hips. ${ }^{24,25}$ A couple of case reports discussed using a large fragment plate for proximal osteotomy and internal lengthening nail for LLD equalisation. ${ }^{26,27}$ To the best of our knowledge, the present study offers a preliminary case series of all-internal PSHR. The application of $3.5 \mathrm{~mm}$ pelvic reconstruction titanium plates instead of a large fragment plate, offered easy contouring and better adaptability when stabilised with screws. The combi-hole design permitted the use of both conventional and locking head screws, thus achieving increased pull out strength. The combination of short and long plates in orthogonal fashion further improved the overall biomechanical strength of the fixation constructs. The introduction of intramedullary lengthening nails created a new milestone in limb-lengthening procedures, particularly in the femur with several advantages. ${ }^{20}$ The reliability of length gain achieved by magnetic lengthening nails was already appreciated. ${ }^{12}$ Also, with the lengthening nail, there is a clear visibility of the regenerate in the standard orthogonal follow-up radiographs compared to external frames, which helps for better decision-making. Due to the limited number of skin incisions which can be closed primarily, lengthening nail offers an improved aesthetic appearance of the limb with no multiple deep scars. Anecdotally, since the utilisation of lengthening nails in place of external fixators, there has been positive feedback from our limb reconstruction nurses and physiotherapists. Also, the senior author (JAF) is currently working on a cost-benefit analysis comparing both lengthening techniques. Another major benefit with internal lengthening nail is that the full range of adjacent joint motion is possible right from the early postoperative period as there are not any transfixing wires/pins through the skin, fascia/ muscles. On the contrary, all the external fixation devices decrease the joint motion even before the commencement of the distraction phase, ${ }^{28}$ thus resulting in prolonged rehabilitation. All that being said, it is important to understand that the PRECICE internal lengthening nail is not a truly load-sharing implant similar to a trauma nail and patients should be cautioned about weight-bearing precautions. 5,20

The PSHR was performed in two stages since it offers an opportunity to plan further with a fresh set of long-axis radiographs, check the availability of internal magnetic lengthening nail and also avoids prolonged anesthesia time (if done as a single stage). It may be feasible to conduct the entire reconstruction in one-stage;

Table 3: Comparison between llizarov hip reconstruction and pelvic support hip reconstruction performed at our institution

\begin{tabular}{lll}
\hline & llizarov hip reconstruction & Pelvic support hip reconstruction \\
\hline Number & 25 & 8 \\
Boys/girls & $16 / 9$ & $4 / 4$ \\
Study period & $2000-2012$ & $2014-2017$ \\
Aetiology & Various & Various \\
Valgus & $51^{\circ}$ & $41^{\circ}$ \\
Extension & $15^{\circ}$ & $15^{\circ}$ \\
Distal varus & $8^{\circ}$ & None \\
Follow-up & 31 months & 19 months \\
Final mechanical axis & 5 mm lateral & 20 mm lateral \\
Length gain & 4.2 cm & 3.5 cm \\
BHI & 50 days/cm & 47 days/cm \\
Pain score $>5$ & 3 & 1 \\
Postoperative Trendelenburg sign & Positive in 7 & Positive in none delayed in 5 \\
Infection & 12 & None \\
Permanent knee stiffness & 2 & None \\
Paley's classification of difficulties in limb & Problems-multiple & Problems-0 \\
lengthening & Obstacles-4 & Obstacles-2 \\
Final result category (as per the & Complications-2 & Complications-0 \\
predesigned scoring system) & $24 \%$ excellent, 28\% good, 24\% fair, & $25 \%$ excellent, 37.5\% good, \\
\hline
\end{tabular}


however, due to the above reasons, the senior author (JAF) felt a staged approach is safer. The patient population of PSHR included diverse pathologies. Femoral head resection was performed in all cases before proximal osteotomy, as described by Milch, 13,29,30 which resulted in satisfactory pain relief with a rapid decline in the pain scores postoperatively. All except one with post-chemotherapy sequelae underwent multiple previous surgeries before the PSHR. The only mechanical complication observed in our series was breakage of both plates at the proximal osteotomy at 8 weeks postsurgery secondary to a mechanical fall. As the patient developed sufficient callus by then, he progressed to a favourable result with no adverse events. No implant-related complications were observed with the PRECICE nails. Trendelenburg sign, positive preoperatively in all eight, turned negative in three patients. The rest of the five demonstrated a delayed response and we postulate this situation to improve with the continuation of physiotherapy and home exercises. It is said to be negative or eliminated if the pelvis on the non-stance side can be elevated high and maintained for 30 seconds. ${ }^{31,32}$ The test is positive if the pelvis on the non-stance cannot be elevated. A delayed positive response means when the pelvis on the non-stance side can be elevated but cannot be held for 30 seconds.

There are certain limitations to our study. It was a retrospective analysis of small sample size with relatively limited follow-up. Weight-bearing precautions during the early postoperative period, in the setting of two osteotomies being stabilised by load-bearing implants together with considerable LLD, should be explained preoperatively. It has been observed, however, that many of our young patients have partially born weight. Further advances in the technology with newer generation lengthening nails may allow immediate weight-bearing. A certain degree of valgisation of the distal femur with lateral deviation of the mechanical axis happens due to femoral lengthening along the anatomical axis. As expected, all patients had a lateral deviation of the mechanical axis at the final follow-up, which needs to be monitored for its long-term effects. One could theorise that deliberate installation of some degree of varus at the distal osteotomy may realign the axis during the lengthening process, something the senior author (JAF) envision in the future. Unlike external fixation, this "all-internal" reconstruction has no scope for postoperative adjustments. Meticulous preoperative planning and strict adherence to the surgical technique, therefore, are of paramount importance for promising and reproducible outcomes. Coming to the evaluation of outcomes, we adopted a scoring system ${ }^{8}$ that was fairly simple and practical, although not a "validated" one. Finally, compared to the external fixators, the magnetic lengthening nail is somewhat more expensive. However, the higher complication rate of fixators, associated with additional hospital stays, medication and physiotherapy sessions finally appear to raise the overall treatment cost.

\section{Conclusion}

In summary, PSHR with exclusive internal devices is a technique in evolution. It avoids common complications of external fixators while facilitating quick rehabilitation of joints, although requires protection from weight-bearing in the early postoperative period. The proposed modification in the surgical technique by refraining from varus at the distal femoral osteotomy can effectively eliminate Trendelenburg gait, albeit with some degree of lateral MAD. Early results are encouraging, however, a bigger study cohort and longer follow-up are necessary to understand the outcomes from a wider perspective.

\section{Clinical Significance}

Pelvic support hip reconstruction performed by all-internal implants is a viable alternative to IHR with potential benefits.

\section{Declarations}

The study was approved by the Ethics and Standard Committee of our institution and the procedures performed were in accordance with the 1964 Helsinki declaration and its later amendments or comparable ethical standards. Informed consent was obtained from all individual participants.

\section{References}

1. Rozbruch SR, Paley D, Bhave A, et al. Ilizarov hip reconstruction for the late sequelae of infantile hip infection. J Bone Jt Surg Am 2005;87(5):1007-1018. DOI: 10.2106/00004623-200505000-00011.

2. Pafilas $D$, Nayagam $S$. The pelvic support osteotomy: Indications and preoperative planning. Strateg Trauma Limb Reconstr 2008;3(2):8392. DOI: 10.1007/s11751-008-0039-7.

3. Ilizarov GA. Hip dislocations. Transosseous Osteosynthesis. Berlin, Heidelberg: Springer; 1992. pp. 701-726.

4. Paley D, Paley D. Hip joint considerations. Principles of Deformity Correction. Berlin, Heidelberg: Springer; 2002. pp. 647-694.

5. Paley D, Herzenberg JE, Paremain G, et al. Femoral lengthening over an intramedullary nail. A matched-case comparison with llizarov femoral lengthening. J Bone Joint Surg Am 1997;79(10):1464-1480. DOI: 10.2106/00004623-199710000-00003.

6. Samchukov ML, Cherkashin AM, Birch JG. Pelvic support osteotomy and limb reconstruction for septic destruction of the hip. Oper Tech Orthop 2013;23(3):158-166. DOI: 10.1053/j.oto.2013.07.009.

7. El-Mowafi H. Outcome of pelvic support osteotomy with the llizarov method in the treatment of the unstable hip joint. Acta Orthop Belg 2005;71(6):686-691.

8. Mahran MA, ElGebeily MA, Ghaly NAM, et al. Pelvic support osteotomy by llizarov's concept: is it a valuable option in managing neglected hip problems in adolescents and young adults? Strateg Trauma Limb Reconstr 2011;6(1):13-20. DOI: 10.1007/s11751-011-01045.

9. Samchukov ML, Birch JG. Pelvic support femoral reconstruction using the method of Ilizarov: a case report. Bull Hosp Jt Dis 1992;52(1):7-11.

10. Paley D. Problems, obstacles, and complications of limb lengthening by the Ilizarov technique. Clin Orthop Relat Res 1990(250):81-104. DOI: 10.1097/00003086-199001000-00011.

11. Fragomen AT, Rozbruch SR. Retrograde magnetic internal lengthening nail for acute femoral deformity correction and limb lengthening. Expert Rev Med Devices 2017;14(10):811-820. DOI: 10.1080/17434440.2017.1378092.

12. Kirane YM, Fragomen AT, Rozbruch SR. Precision of the $\mathrm{PRECICE}^{\oplus}$ internal bone lengthening nail. Clin Orthop Relat Res 2014;472(12):3869-3878. DOI: 10.1007/s11999-014-3575-0.

13. Milch $\mathrm{H}$. The resection-angulation operation for hip-joint disabilities. J Bone Joint Surg Am 1955;37(4):699-717. DOI: 10.2106/00004623195537040-00004.

14. Moras P, Belthur MV, Jones SJFJ. Pelvic support osteotomy - Revisited. Orthop Proc 2006;88-B(SUPP_III):436.

15. Nunn TR, Pratt E, Dickens W, et al. Ilizarov hip reconstruction for the adolescent difficult problem hip. Orthop Proc 2013;95(SUPP_23):24.

16. Hasler CC, Krieg AH. Current concepts of leg lengthening. J Child Orthop 2012;6(2):89-104. DOI: 10.1007/s11832-012-0391-5.

17. Green SA. Complications of external skeletal fixation. Clin Orthop Relat Res 1983(180):109-116. DOI: 10.1097/00003086-19831100000015. 
18. Singh $S$, Lahiri $A$, lqbal $M$. The results of limb lengthening by callus distraction using an extending intramedullary nail (Fitbone) in nontraumatic disorders. J Bone Joint Surg Br 2006;88(7):938-942. DOI: 10.1302/0301-620X.88B7.17618.

19. Krieg $A H$, Speth BM, Foster BK. Leg lengthening with a motorized nail in adolescents an alternative to external fixators? Clin Orthop Relat Res 2008;466(1):189-197. DOI: 10.1007/s11999-007-0040-3.

20. Baumgart R, Betz A, Schweiberer L. A fully implantable motorized intramedullary nail for limb lengthening and bone transport. Clin Orthop Relat Res 1997;343:135-143. DOI: 10.1097/00003086199710000-00023.

21. Laubscher M, Mitchell C, Timms A, et al. Outcomes following femoral lengthening: an initial comparison of the precice intramedullary lengthening nail and the LRS external fixator monorail system. Bone Jt J 2016;98-B(10):1382-1388. DOI: 10.1302/0301-620X.98B10. 36643.

22. Barker KL, Simpson AH, Lamb SE. Loss of knee range of motion in leg lengthening. J Orthop Sports Phys Ther 2001;31(5):238-244. DOI: 10.2519/jospt.2001.31.5.238discussion 245-6.

23. Glorion C, Pouliquen JC, Langlais J, et al. Femoral lengthening using the callotasis method: study of the complications in a series of 70 cases in children and adolescents. J Pediatr Orthop 16(2):161-167. DOI: 10.1097/01241398-199603000-00005.

24. Hogan KA, Blake M, Gross RH. Subtrochanteric valgus osteotomy for chronically dislocated, painful spastic hips. J Bone Jt Surg - Ser A 2006;88(12):2624-2631. DOI: 10.2106/00004623-20061200000009 .
25. Aksoy MC, Musdal Y. Subtrochanteric valgus-extension osteotomy for neglected congenital dislocation of the hip in young adults. Acta Orthop Belg 2000;66(2):181-186.

26. Krieg $\mathrm{AH}$, Lenze $\mathrm{U}$, Hasler CC. Ilizarov hip reconstruction without external fixation: a new technique. J Child Orthop 2010;4(3):259-266. DOI: 10.1007/s11832-010-0256-8.

27. Reynders-Frederix P, Reynders-Frederix C, Wajid M. Pelvic support osteotomy; salvage procedure in chronically dislocated hips case report: technical note on the on-lay bone positioning of a lengthening nail and review of the literature. Open Orthop J 2016;10(1):232-240. DOI: 10.2174/1874325001610010232.

28. Maffulli N, Nele U, Matarazzo L. Changes in knee motion following femoral and tibial lengthening using the llizarov apparatus: a cohort study. J Orthop Sci 2001;6(4):333-338. DOI: 10.1007/s007760100028.

29. Milch H. Surgical treatment of the stiff, painful hip--the resectionangulation operation. Clin Orthop Relat Res 1963;31(1):48-57. DOI: 10.1097/00003086-196300310-00007.

30. Brand RA. 50 Years ago in CORR: technic of the resection-angulation operation for hip-joint disabilities. henry milch MD CORR;13:265-270. Clin Orthop Relat Res 2010;468(2):628-630. DOI: 10.1007/s11999-0091155-5.

31. Bailey R, Selfe J, Richards J. The role of the Trendelenburg test in the examination of gait. Phys Ther Rev 2009;14(3):190-197. DOI: $10.1179 / 174328809 \times 452836$.

32. Hardcastle $P$, Nade $S$. The significance of the Trendelenburg test. J Bone Joint Surg Br 1985;67(5):741-746. DOI: 10.1302/0301620X.67B5.4055873. 